\title{
Screening a Protein Array with Synthetic Biotinylated Inorganic Polyphosphate To Define the Human PolyP-ome
}

\author{
Cristina Azevedo, ${ }^{\dagger}$ Jyoti Singh, ${ }^{\S}$ Nicole Steck, ${ }^{\S}$ Alexandre Hofer, ${ }^{\|}$Felix A. Ruiz, ${ }^{\dagger \neq}$ Tanya Singh, ${ }^{\dagger}$ \\ Henning J. Jessen, $*, \$ \odot$ and Adolfo Saiardi $*, \odot \odot$

\begin{abstract}
${ }^{\dagger}$ Medical Research Council Laboratory for Molecular Cell Biology, University College London, London, WC1E 6BT, United Kingdom

${ }^{\ddagger}$ Research Unit, "Puerta del Mar” University Hospital, School of Medicine, and Institute of Biomedical Research Cadiz (INiBICA), University of Cadiz, Cadiz, Spain

${ }^{\S}$ Institute of Organic Chemistry, University of Freiburg, Albertstrasse 21, 79104 Freiburg, Germany

"Department of Chemistry, University of Zurich, Winterthurerstrasse 190, 8057 Zurich, Switzerland
\end{abstract}

\section{Supporting Information}

ABSTRACT: Phenotypes are established by tight regulation on protein functions. This regulation can be mediated allosterically, through protein binding, and covalently, through post-translational modification (PTM). The integration of an ever-increasing number of PTMs into regulatory networks enables and defines the proteome complexity. Protein PTMs can occur enzymatically and nonenzymatically. Polyphosphorylation, which is a recently discovered PTM that belongs to the latter category, is the covalent attachment of the linear ortho-phosphate polymer called inorganic polyphosphate (polyP) to lysine residues. PolyP, which is ubiquitously present in nature, is also known to allosterically control

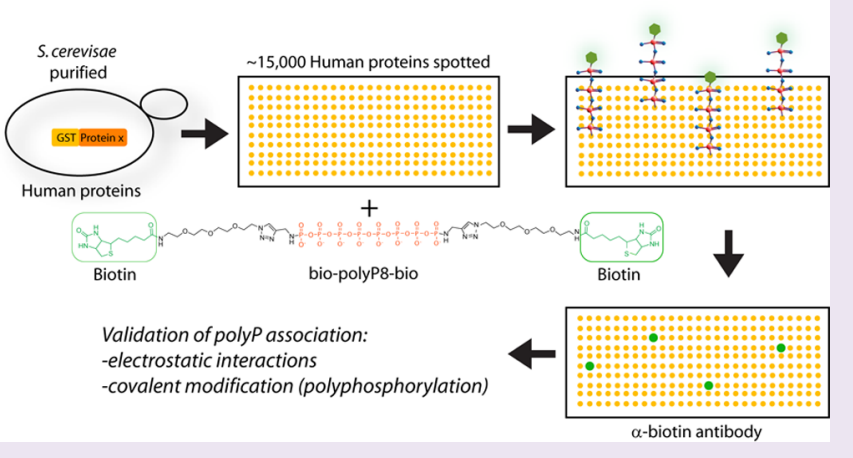
protein function. To date, lack of reagents has prevented the systematic analysis of proteins covalently and/or allosterically associated with polyP. Here, we report on the chemical synthesis of biotin-modified monodisperse short-chain polyP (bio-polyP8-bio) and its subsequent use to screen a human proteome array to identify proteins that associate with polyP, thereby starting to define the human polyP-ome.

$\mathrm{T}^{\mathrm{k}}$ he human proteome is much more complex than previously recognized, with an ever-increasing number of protein post-translational modifications (PTMs) being identified. $^{1-3}$ Some PTMs are introduced enzymatically, while others are not dependent on enzymes. ${ }^{2}$ Polyphosphorylation is a newly discovered nonenzymatic PTM in which the linear polymer inorganic polyphosphate (polyP), which is solely composed of condensed phosphates, is covalently attached to lysine residues through a phosphoramidate $\mathrm{P}-\mathrm{N}$ bond. ${ }^{4,5}$ This modification results from a nucleophilic attack by a lysine residue of the target protein to an internal phosphate of the polyP chain. ${ }^{5}$ The number of orthophosphate monomers in polyP ranges from tens to thousands, making this modification polydisperse or nonuniform. So far, the acid lability of the phosphoramidate functional group and the polymeric nature of polyphosphorylation has prevented its detection by mass spectrometry. ${ }^{4,6}$ Characterization of polyphosphorylation has only been possible in Saccharomyces cerevisiae and through extensive biochemical examinations. The structural determinant for polyphosphorylation resides in a specific cluster designated the PASK domain [Poly-Acidic serine $(S)$ and lysine $(K)$ rich.$^{5}$ However, the required extension of this domain and the importance of each residue for the modification are unknown. Two polyphosphorylation targets were originally identified: Nuclear signal recognition 1 (Nsr1) and its interacting partner Topoisomerase 1 (Top1). Recently, 15 more yeast-validated targets have been identified through bioinformatics analysis. ${ }^{7}$

Inorganic polyphosphate is present in all domains of life: bacteria, archaea, and eukaryotes. ${ }^{8,9}$ In mammals, polyP plays various roles including acting as a hemostasis regulator, ${ }^{10-13}$ as a nucleating factor for fibril formation, ${ }^{14}$ and is required for bone mineralization. ${ }^{15}$ The molecular mechanisms by which polyP exerts these functions are not understood but are likely to be related to its ability to associate with specific client proteins, either through ionic interactions ${ }^{16-18}$ or through covalent protein modifications such as polyphosphorylation. ${ }^{5}$

Here, we report an approach to systematically identify proteins that associate with polyP in mammals. We chemically synthesized a polyP species of defined size, eight phosphates in

Received: April 18, 2018

Accepted: June 20, 2018

Published: June 20, 2018 


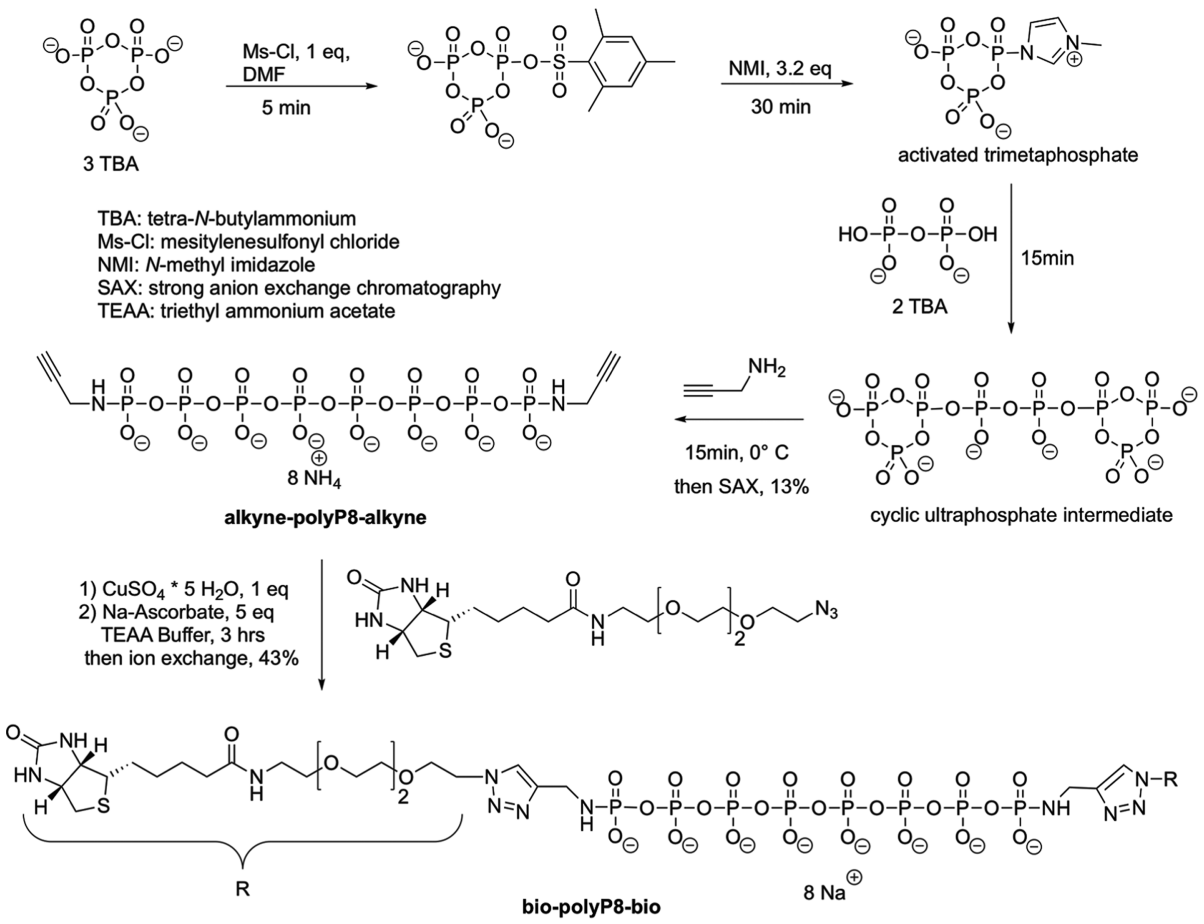

Figure 1. Synthesis of bio-polyP8-bio by bidirectional extension. Activation of cyclic trimetaphosphate with mesitylenesulfonyl chloride (Ms-Cl) and $\mathrm{N}$-methylimidazole results in activated trimetaphosphate, which can react with pyrophosphate at both termini to give a cyclic ultraphosphate intermediate. This intermediate is ring-opened with propargylamine. All reactions occur in one flask. Next, the alkyne-polyP8-alkyne is click-labeled with biotin, using the Huisgen dipolar cycloaddition.

length, that is capped at both ends with biotin (bio-polyP8bio). This chemical tool was used in a human proteome array to identify proteins that associate electrostatically and covalently with polyP, thereby defining the "polyP-ome".

The synthesis of monodisperse polyP building blocks has been an unsolved problem and only the modification of polydisperse polyP has been reported. ${ }^{19,20}$ Here, we present a bottom-up approach that enables the synthesis of short oligophosphates with a defined composition (Figure 1). This represents the first report on a bidirectional extension of a diphosphate unit with activated trimetaphosphate followed by ring-opening with amine nucleophiles. This strategy enabled the modification of a P8 stretch with P-amidates at the terminal positions introducing an alkyne. The bis-alkyne was used in a 2 -fold Huisgen cycloaddition reaction ${ }^{21}$ to install biotin labels at both ends. In brief, trimetaphosphate was activated with mesitylenesulfonyl chloride and $N$-methylimidazole $^{22}$ and reacted with substoichiometric amounts of pyrophosphate. The formation of a cyclic ultraphosphate at both termini was analyzed by ${ }^{31} \mathrm{P}$ NMR [see Figure $\mathrm{S} 1$ (spectrum 10) in the Supporting Information] and the expected coupling pattern for the different phosphate units was observed ( $d$, dd, and $d t)$. The intermediate was ringopened by the addition of propargylamine and linearization occurred. The material was purified by ion exchange chromatography on Q-Sepharose with an ammonium bicarbonate gradient and buffer was removed by lyophilization. NMR analysis revealed that the terminal P-amidates resonated at ca. $-2 \mathrm{ppm}$ and the internal anhydrides at $-23 \mathrm{ppm}$ with a ratio of 2:6 as expected [see Figure S1 (spectrum 2)].

The identity of the material was further confirmed by highresolution $\mathrm{MS}$ and $2 \mathrm{D}$ NMR spectroscopy $\left({ }^{31} \mathrm{P}-{ }^{1} \mathrm{H}\right.$ HMBC and ${ }^{31} \mathrm{P}-{ }^{31} \mathrm{P}$ COSY) giving the correct cross peak pattern.
Overall, the construction of a monodisperse P8 unit with modified termini was achieved in a one-flask reaction with an overall yield of $13 \%$. The alkynylated polyP 8 was then subjected to azide-alkyne cycloaddition introducing the biotin labels at both ends. The product precipitated from solution by addition of sodium iodide/acetone and the sodium salt of biopolyP8-bio was collected in $43 \%$ yield.

To identify the human polyP-ome, we screened a commercially available Human Proteome Array slide, containing over 15000 human proteins expressed and purified from yeast, with bio-polyP8-bio. In 2004, Nomura et al. demonstrated that ${ }^{32} \mathrm{P}$-labeled polyP could be specifically transferred to proteins blotted onto membranes. ${ }^{23}$ Similarly, the transfer of biotin to recombinant His-Top1(PASK) can be detected upon incubation with bio-polyP8-bio (Figure S2A in the Supporting Information). Moreover, we could detect transfer of polyP to the protein when $1 \mu \mathrm{M}$ of bio-polyP8-bio was incubated with $0.5 \mu \mathrm{g}$ His-Top1(PASK) (Figure S2B in the Supporting Information), demonstrating the validity of bio-polyP8-bio as a screening tool to identify polyP clients. Since the exact amount of protein spotted onto the glass chip is unknown and the substrate (glass) is different from nitrocellulose, $20 \mu \mathrm{M}$ of biopolyP8-bio were used in the initial screen (Screen A). Under these conditions, 309 proteins were detected with a $1.3 \mathrm{Hit}$ score (HS) cutoff above background, corresponding to $2 \%$ of the library (see Table S1 in the Supporting Information).

We expected to detect targets that associate with polyP through either electrostatic or covalent interactions. To distinguish between these, we relied on a protein mobility shift observed on Bis-Tris Gels/NuPAGE ${ }^{5}$ for covalent polyphosphorylation. Conversely, for proteins that interact with polyP through ionic interactions, no mobility shift on BisTris Gels/NuPAGE should be expected. However, a shift 

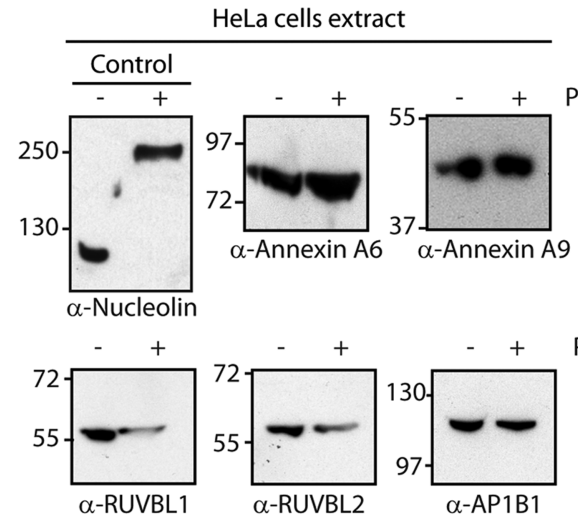

C.
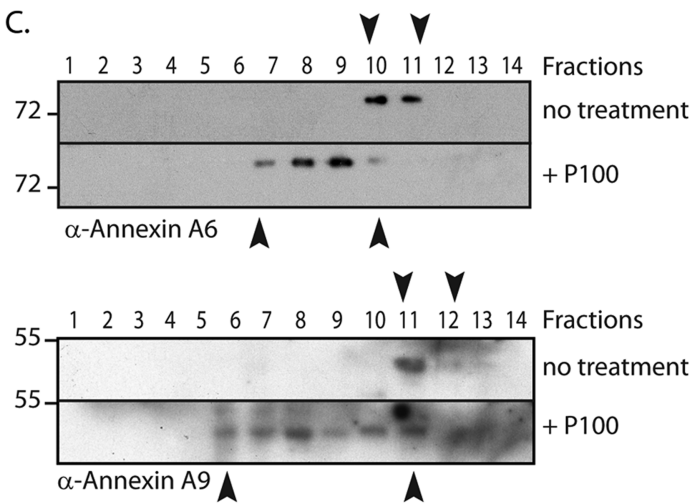

E.

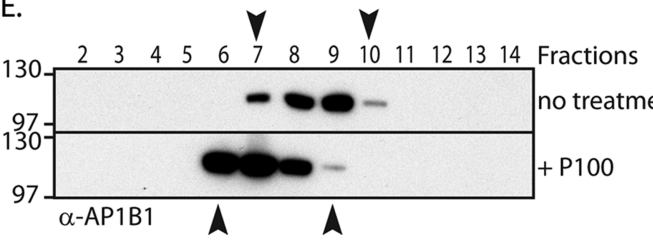

B.

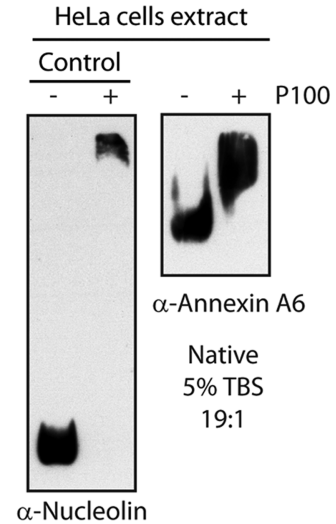

D.

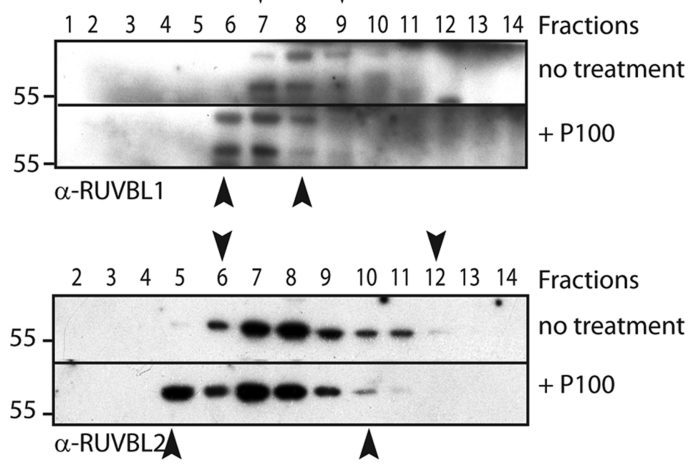

F.

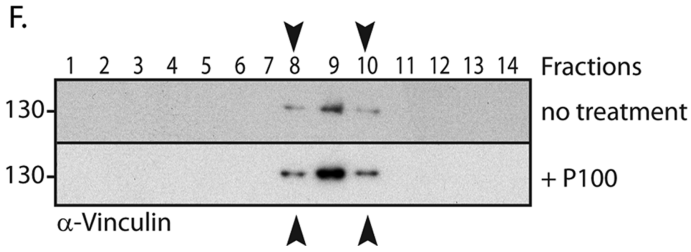

Figure 2. (A) Annexin A6 and Annexin A9, RUVBL1 and RUVBL2 and AP1B1 are not polyphosphorylated. HeLa cell protein extracts untreated $(-)$ or treated $(+)$ with polyP with an average size of 100 phosphate residues (P100) were resolved by Bis-Tris Gels/NuPAGE and immunoblotted. (B) Annexin A6 associates with polyP electrostatically; HeLa native protein extracts, untreated or treated with P100, were resolved by 5\% TBS 15:1 native PAGE and immunoblotted. (C) Annexin A6 and Annexin A9, (D) RUVBL1 and RUVBL 2 and (E) AP1B1 associate with polyP electrostatically; HeLa cell native protein extracts, untreated or treated with P100, were resolved by gel filtration (Superdex200 Increase 10/ 300GL) and immunoblotted. (F) Vinculin does not associate with polyP; incubation of HeLa cell extracts with P100 does not change the gel filtration elution profile. The data are representative of three independent repeats producing similar results.

should be observed under native PAGE conditions. In budding yeast, an organism containing high levels of poly $\mathrm{P}^{24,25}$ and in which the polyP biosynthetic pathway is characterized, it is possible to study the mobility shift of proteins in vivo, comparing between mutants with altered polyP metabolism. ${ }^{26}$ In mammalian cells, this direct assay is not possible because the concentration of polyP is much lower, ${ }^{27}$ and the enzymology of its synthesis is unknown. For this, we relied on an indirect approach: polyP was added exogenously to mammalian protein extracts before assaying the mobility shift. As a positive control, we used the human homologue of the yeast target Nsr1, nucleolin, that possesses a striking PASK domain (see Figure S3A in the Supporting Information). To confirm nucleolin as a target, we expressed it in wild-type yeast. GST-nucleolin has a smeary appearance with a molecular weight that is greater than its predicted size of $77 \mathrm{kDa}$ (see Figure S3B in the Supporting Information). In vtc $4 \Delta$, a yeast with impaired polyP synthesis, GST-nucleolin runs as a single band of lower molecular weight. Moreover, exogenously incubating vtc $4 \Delta$ extracts with polyP results in mobility shift (see Figure S3B). Similarly, in HeLa cell extracts, endogenous nucleolin is shifted upward upon polyP treatment (see Figure $\mathrm{S} 3 \mathrm{C}$ in the Supporting Information, as well as Figure 2A). Together, these data indicate that nucleolin is a polyphosphorylation target. The following criteria were used to select the screening hits to authenticate: molecular weight between 40 and $110 \mathrm{kDa}$, expressed in HeLa, and for which an antibody is available. Furthermore, preference was given to proteins belonging to a family of which more than one member gave a positive hit. To avoid bias, the protein sequence itself was not taken into consideration (i.e., presence of PASK domain). Based on these criteria, we validated 11 hits (see Table 1).

We observed that Annexin A6 and A9, FAF1, DPP3, RUVBL1 and RUVBL2, vinculin, and AP1B1 did not show a mobility shift on Bis-Tris Gels/NuPAGE, suggesting that, if there is association with polyP, it is through electrostatic interaction (see Figure 2A and Figure S4A in the Supporting Information). To test this hypothesis, we investigated their mobility under native conditions. FAF1 and DPP3 also failed to show a mobility shift under native conditions, suggesting 
Table 1. Validated Hits from Bio-polyP8-bio Screens

\begin{tabular}{|c|c|c|c|}
\hline protein & gene name/uniprot & description & $\begin{array}{l}\text { validation } \\
\text { result }\end{array}$ \\
\hline \multicolumn{4}{|c|}{ Screen A } \\
\hline Annexin A6 & ANXA6/P08133 & calcium-dependent phospholipids binding protein family member & electrostatic \\
\hline Annexin A9 & ANXA9/O76027 & as above & electrostatic \\
\hline RuvB-like 1 & RUVBL1/Q9Y265 & $\begin{array}{l}\text { single-stranded DNA-stimulated ATPase and ATP-dependent DNA helicase ( } 5^{\prime} \\
\left.\text { to } 3^{\prime}\right) \text { activity }\end{array}$ & electrostatic \\
\hline RuvB-like 2 & RUVBL2/Q9Y230 & as above & electrostatic \\
\hline FAS-associated factor 1 & FAF1/Q9UNN5 & $\begin{array}{l}\text { multidomain protein regulating apoptosis, ubiquitination and proteasomal } \\
\text { degradation }\end{array}$ & false positive \\
\hline dipeptidyl-peptidase 3 & DPP3/Q9NY33 & $\begin{array}{l}\text { broad spectrum aminopeptidases that cleaves dipeptides from the } \mathrm{N} \text {-terminal of } \\
\text { proteins }\end{array}$ & false positive \\
\hline vinculin & VCL/P18206 & cytoskeletal protein associated with cell-cell and cell-matrix junctions & false positive \\
\hline tetratricopeptide repeat protein 27 & TTC27/Q6P3X3 & $\begin{array}{l}\text { TPR domains mediate protein-protein interactions and the assembly of } \\
\text { multiprotein complexes }\end{array}$ & covalent \\
\hline endoplasmin & HSP90B1/P14625 & endoplasmic reticulum protein of heat shock protein 90 family. & covalent \\
\hline $\begin{array}{l}\text { translation initiation factor eIF-2B subunit } \\
\text { epsilon }\end{array}$ & EIF2B5/Q13144 & catalyzes the exchange of eukaryotic initiation factor 2-bound GDP for GTP & covalent \\
\hline \multicolumn{4}{|c|}{ Screen B } \\
\hline Gelsolin & GSN/P06396 & $\begin{array}{l}\text { actin-associated protein promoting the assembly of actin monomers into } \\
\text { filaments }\end{array}$ & covalent \\
\hline general transcription factor II-I & GTF2I/P78347 & component of the basal transcription machinery & covalent \\
\hline eyes absent homologue 1 & EYA1/Q99502 & $\begin{array}{l}\text { dual activity enzyme- phosphatase and transcriptional coactivator for some of } \\
\text { the SIX proteins }\end{array}$ & covalent \\
\hline
\end{tabular}

that these proteins do not associate with polyP and are false positives (Figure S4B in the Supporting Information). Annexin A6 showed a mobility shift under native conditions, suggesting that there is an electrostatic association with polyP (see Figure 2B). Annexin A9, vinculin, AP1B1, and RUVBL1 and RUVBL2 antibodies did not recognize the native form of the proteins. To overcome this, we performed gel filtration experiments. Gel filtration analysis of annexin A6 and A9, RUVBL1 and RUVBL2, and AP1B1 in HeLa protein extracts treated with polyP showed that these proteins elute in earlier fractions than untreated proteins, indicating an increase in size as a direct consequence of polyP association or through polyP-dependent interaction with partners (Figures 2C-E). Vinculin, on the other hand, elutes in the same fractions with or without polyP treatment, indicating that this protein, such as FAF1 and DPP3, does not associate with polyP (Figure 2F). TTC27, HSP90B1, and eIF2B epsilon showed a clear mobility shift on Bis-Tris Gels/NuPAGE, suggesting that they are covalent polyphosphorylation targets (see Figure 3A).

Our validation revealed that vinculin, FAF1, and DPP3 are false positives. Therefore, we performed a higher stringency screen by decreasing the amount of bio-polyP8-bio 4-fold (Screen B). In doing so, we approximately halved the total number of hits obtained (155 proteins), which corresponds to $\sim 1 \%$ of the library (Table S2 in the Supporting Information). Overlap analysis showed that $50 \%$ of the proteins (i.e., 75 hits) are common between the screens (see Figure S5 and Table S3 in the Supporting Information). This variance is to be expected, because of the difference in the protocol (with regard to materials and methods) and the different batch of screening slides. We validated three additional proteins that were present only in screen B: Gelsolin, GTF2i, and EYA1 (Table 1). Gel shift assays with antibodies specific for these proteins demonstrated that they all show decreased mobility upon treatment with polyP (Figure 3B), suggesting that these proteins are polyphosphorylated. Annexin A6, A9, TTC27, RUVBL1, and HSP90B1 were among the common proteins
A.

HeLa cells extract

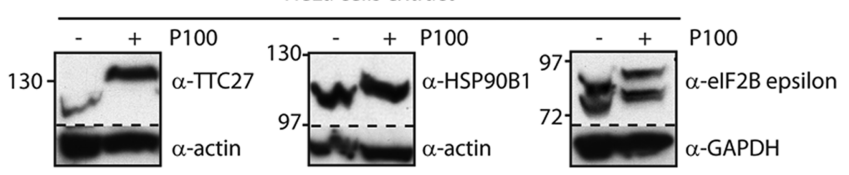

B.

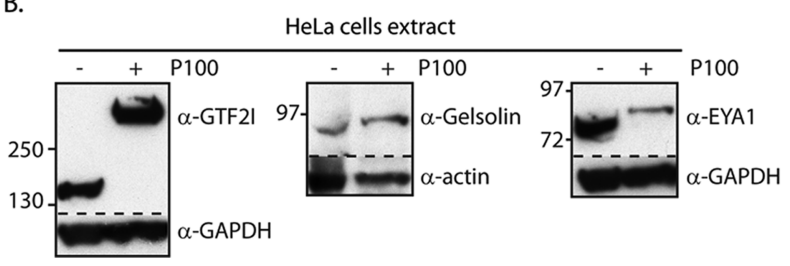

Figure 3. Novel polyphosphorylation targets validated from biopolyP8-bio screens A and B. The hits TTC27, HSP90B1, and eIF2B epsilon from Screen A (panel (A)) as well as the hits GTF2I, gelsolin, and EYAl from Screen B (panel (B)) are polyphosphosphorylated. HeLa cell protein extracts untreated or treated with P100 were resolved by Bis-Tris Gels/NuPAGE gel and immunoblotted. Four independent repeats produced similar results. Stroke lines are used when the figure was assembled from the same gel/membrane.

between the screens. Importantly, neither vinculin, FAF1, nor DPP3 were present in the higher stringency screen, suggesting that we succeeded in reducing the number of false positives. Bioinformatics analysis of the combined screens (see the Methods section in the Supporting Information) did not reveal an enrichment of any particular protein family or protein domain. However, the analysis of the gene ontology (GO) biological process revealed an enrichment of proteins involved in metabolic processes and, specifically, in amino acid biosynthesis (see Figure S6 in the Supporting Information).

Until now, we could only identify polyphoshorylation targets that have striking PASK domains such as Nsr1, Top1, and Nucleolin, and, to characterize them, we solely relied on an Bis-Tris Gels/NuPAGE gel mobility shift, which is very prominent for these proteins. However, sequence analysis of 
the confirmed hits from both screens revealed that, with this new method, we managed to identify proteins, such as TTC27, eIF2B, gelsolin, and EYA1, with very subtle PASK domains and, consequently, less-evident shifts. Indeed, a recently published bioinformatics screen looking for proteins with prominent PASK-domains (one or more 20 amino acid stretches composed of at least $75 \% \mathrm{D} / \mathrm{E} / \mathrm{S}$ and at least one $\mathrm{K})$ reported yeast and mammalian targets, ${ }^{7}$ with little overlap with our hits. The reason our screen did not identify hits with striking PASK domains resides in the fact that, when expressed and purified from yeast, which is an organism with a very high amount of polyP, ${ }^{24}$ these proteins could already be associated with polyP and are therefore less likely to be identified in this screen. An example is nucleolin, identified as a polyphosphorylation target based on its homology with the yeast Nsr1, but was not a hit in our screens. When expressed in WT yeast, nucleolin showed a very high mobility shift (Figure S3B in the Supporting Information) suggesting that, when purified, this protein is already highly polyphosphorylated, preventing it from further modification with bio-polyP8-bio. In contrast, proteins with subtle PASK domains are likely to lose the association with yeast polyP upon purification, because of the known post-extraction instability of polyphosphorylation, and could therefore associate with bio-P8-bio.

Some of the identified polyP targets are members of conserved protein families. To expand the polyP-ome to other family members, we performed sequence analysis on some of the targets. Analysis of HSP90B1 revealed the presence of a possible PASK domain within the "charged linker" region connecting the $\mathrm{N}$-terminus to the middle domain (Figure S7A in the Supporting Information). This region is conserved in HSP90B1's cytosolic counterparts, HSP90 $\alpha / \beta$ (Figure S7B in the Supporting Information). We tested if HSP $90 \alpha / \beta$ could also be polyphosphorylated. Upon polyP treatment, cytosolic HSP90s (HSP90 $\alpha / \beta)$ show a significant mobility shift on BisTris Gels/NuPAGE gels (Figure S7C in the Supporting Information). Similarly, sequence analysis of AP1B1 revealed a region at the $\mathrm{N}$-terminus, within the head domain, with a few lysine and acidic amino acids (Figures S8A and S8B in the Supporting Information). This sequence is conserved in AP1B1's closest homologue AP2B1, but not in AP3B1. However, AP3B1, which is a protein previously shown to be pyrophosphorylated on serine residues, ${ }^{28}$ contains two further regions with strong PASK domain similarity: one within the head and the other within the hinge domains (see Figures S8A and $\mathrm{S} 8 \mathrm{~B})$. We tested if AP3B1 could be a target of polyphosphorylation. Myc-AP3B1 showed a mobility shift on NuPAGE upon incubation of transfected HeLa protein extracts with polyP (see Figure S8C). This result suggests that, unlike that observed for AP1B1, the association of AP3B1 with polyP is covalent.

In conclusion, the original use of bio-polyP8-bio has allowed us to start defining the mammalian polyP-ome and, more importantly, will help us to start defining the minimal PASK domain. We identified a few hundred putative polyP-associated targets with subtle PASK domains necessary for polyphosphorylation. This, however, is likely to still represent an underestimation of the total number of targets, since the protein microchip library available lacks $1 / 4$ of the human proteome. Our work confirms that proteins interact with polyP via two distinct modes of action: electrostatic association and covalent modification. Lysine polyphosphorylation as a covalent PTM has only been described very recently, and, thus far, only a handful of proteins have been reported to associate with polyP electrostatically. ${ }^{16,17}$ It will be important to study the precise nature of the electrostatic interactions between polyP and proteins and its regulation.

The bio-polyP8-bio synthesized here (recall Figure 1) consists of only a relatively short polyP chain. However, this new method will enable the bidirectional synthesis of muchlonger condensed phosphates, which might help identify additional polyphosphorylation targets, since it is possible that the polyP chain length determines substrate specificity. Moreover, the use of click chemistry will provide useful tools to construct a variety of other compounds, such as fluorescently tagged defined polyP. The defined mass of biopolyP8-bio make it a versatile tool for polyP research that will facilitate the identification of polyphosphorylated lysine residues, since only a restricted range of added mass with different charge states must be analyzed, bypassing one of the main problems that arises from the polymeric nature of this and other PTMs, namely, the unknown size of the modification.

\section{METHODS}

All methods are described in the Supporting Information.

\section{ASSOCIATED CONTENT}

\section{S Supporting Information}

The Supporting Information is available free of charge on the ACS Publications website at DOI: 10.1021/acschembio.8b00357.

Materials, methods, and supplementary figures (PDF)

A detailed description of the synthetic procedures (PDF)

\section{AUTHOR INFORMATION}

\section{Corresponding Authors}

*E-mail: henning.jessen@oc.uni-freiburg.de (H. J. Jessen). *E-mail: dmcbado@ucl.ac.uk (A. Saiardi).

ORCID

Cristina Azevedo: 0000-0002-6997-1265

Henning J. Jessen: 0000-0002-1025-9484

Adolfo Saiardi: 0000-0002-4351-0081

\section{Notes}

The authors declare no competing financial interest.

\section{ACKNOWLEDGMENTS}

The authors thank the members of the Jessen and Saiardi Laboratories. This work was supported by the Medical Research Council (MRC) core support to the MRC/UCL Laboratory for Molecular Cell Biology University Unit, MC U12266B to T.S. (Bioinformatics Image Core) and MC_UU_1201814 to C.A. and A.S. and an HFSP program grant (RP $\mathrm{P} 0025 / 2016)$ to J.S., N.S., and H.J. F.R. was the recipient of a scholarship grant from the Government of Spain ("Salvador de Madariaga” program MECD PRX17/00473).

\section{REFERENCES}

(1) Azevedo, C., and Saiardi, A. (2016) The new world of inorganic polyphosphates. Biochem. Soc. Trans. 44, 13-17.

(2) Harmel, R., and Fiedler, D. (2018) Features and regulation of non-enzymatic post-translational modifications. Nat. Chem. Biol. 14, 244-252. 
(3) Walsh, C. T., Garneau-Tsodikova, S., and Gatto, G. J. (2005) Protein posttranslational modifications: the chemistry of proteome diversifications. Angew. Chem., Int. Ed. 44, 7342-7372.

(4) Azevedo, C., and Saiardi, A. (2016) Why always lysine? The ongoing tale of one of the most modified amino acids. Adv. Biol. Regul $60,144-150$.

(5) Azevedo, C., Livermore, T., and Saiardi, A. (2015) Protein polyphosphorylation of lysine residues by inorganic polyphosphate. Mol. Cell 58, 71-82.

(6) Besant, P. G., Attwood, P. V., and Piggott, M. J. (2009) Focus on phosphoarginine and phospholysine. Curr. Protein Pept. Sci. 10, 536550 .

(7) Bentley-DeSousa, A., Holinier, C., Moteshareie, H., Tseng, Y. C., Kajjo, S., Nwosu, C., Amodeo, G. F., Bondy-Chorney, E., Sai, Y., Rudner, A., Golshani, A., Davey, N. E., and Downey, M. (2018) A screen for candidate targets of lysine polyphosphorylation uncovers a conserved network implicated in ribosome biogenesis. Cell Rep. 22, 3427-3439.

(8) Morrissey, J. H., Choi, S. H., and Smith, S. A. (2012) Polyphosphate: an ancient molecule that links platelets, coagulation, and inflammation. Blood 119, 5972-5979.

(9) Jiménez, J., Bru, S., Ribeiro, M. P., and Clotet, J. (2017) Polyphosphate: popping up from oblivion. Curr. Genet. 63, 15-18.

(10) Azevedo, C., and Saiardi, A. (2014) Functions of inorganic polyphosphates in eukaryotic cells: a coat of many colours. Biochem. Soc. Trans. 42, 98-102.

(11) Morrissey, J. H., and Smith, S. A. (2015) Polyphosphate as modulator of hemostasis, thrombosis, and inflammation. J. Thromb. Haemostasis 13, S92-S97.

(12) Ruiz, F. A., Lea, C. R., Oldfield, E., and Docampo, R. (2004) Human platelet dense granules contain polyphosphate and are similar to acidocalcisomes of bacteria and unicellular eukaryotes. J. Biol. Chem. 279, 44250-44257.

(13) Smith, S. A., Mutch, N. J., Baskar, D., Rohloff, P., Docampo, R., and Morrissey, J. H. (2006) Polyphosphate modulates blood coagulation and fibrinolysis. Proc. Natl. Acad. Sci. U. S. A. 103, 903-908.

(14) Cremers, C. M., Knoefler, D., Gates, S., Martin, N., Dahl, J. U., Lempart, J., Xie, L., Chapman, M. R., Galvan, V., Southworth, D. R., and Jakob, U. (2016) Polyphosphate: A conserved modifier of amyloidogenic processes. Mol. Cell 63, 768-780.

(15) Omelon, S., Ariganello, M., Bonucci, E., Grynpas, M., and Nanci, A. (2013) A review of phosphate mineral nucleation in biology and geobiology. Calcif. Tissue Int. 93, 382-396.

(16) Mutch, N. J., Myles, T., Leung, L. L., and Morrissey, J. H. (2010) Polyphosphate binds with high affinity to exosite II of thrombin. J. Thromb. Haemostasis 8, 548-555.

(17) Zakharian, E., Thyagarajan, B., French, R. J., Pavlov, E., and Rohacs, T. (2009) Inorganic polyphosphate modulates TRPM8 channels. PLoS One 4, e5404.

(18) Montilla, M., Hernández-Ruiz, L., García-Cozar, F. J., AlvarezLaderas, I., Rodríguez-Martorell, J., and Ruiz, F. A. (2012) Polyphosphate binds to human von Willebrand factor in vivo and modulates its interaction with glycoprotein Ib. J. Thromb. Haemostasis $10,2315-2323$.

(19) Kudela, D., Smith, S. A., May-Masnou, A., Braun, G. B., Pallaoro, A., Nguyen, C. K., Chuong, T. T., Nownes, S., Allen, R., Parker, N. R., Rashidi, H. H., Morrissey, J. H., and Stucky, G. D. (2015) Clotting activity of polyphosphate-functionalized silica nanoparticles. Angew. Chem., Int. Ed. 54, 4018-4022.

(20) Choi, S. H., Collins, J. N., Smith, S. A., Davis-Harrison, R. L., Rienstra, C. M., and Morrissey, J. H. (2010) Phosphoramidate end labeling of inorganic polyphosphates: facile manipulation of polyphosphate for investigating and modulating its biological activities. Biochemistry 49, 9935-9941.

(21) Kolb, H. C., Finn, M. G., and Sharpless, K. B. (2001) Click Chemistry: Diverse Chemical Function from a Few Good Reactions. Angew. Chem., Int. Ed. 40, 2004-2021.
(22) Mohamady, S., and Taylor, S. D. (2013) Synthesis of nucleoside tetraphosphates and dinucleoside pentaphosphates via activation of cyclic trimetaphosphate. Org. Lett. 15, 2612-2615.

(23) Nomura, K., Kato, J., Takiguchi, N., Ohtake, H., and Kuroda, A. (2004) Effects of inorganic polyphosphate on the proteolytic and DNA-binding activities of Lon in Escherichia coli. J. Biol. Chem. 279, 34406-34410.

(24) Kornberg, A., Rao, N. N., and Ault-Riché, D. (1999) Inorganic polyphosphate: a molecule of many functions. Annu. Rev. Biochem. 68, 89-125.

(25) Rao, N. N., Gómez-García, M. R., and Kornberg, A. (2009) Inorganic polyphosphate: essential for growth and survival. Annu. Rev. Biochem. 78, 605-647.

(26) Brown, M. R., and Kornberg, A. (2004) Inorganic polyphosphate in the origin and survival of species. Proc. Natl. Acad. Sci. U. S. A. 101, 16085-16087.

(27) Kumble, K. D., and Kornberg, A. (1995) Inorganic polyphosphate in mammalian cells and tissues. J. Biol. Chem. 270, $5818-5822$

(28) Azevedo, C., Burton, A., Ruiz-Mateos, E., Marsh, M., and Saiardi, A. (2009) Inositol pyrophosphate mediated pyrophosphorylation of AP3B1 regulates HIV-1 Gag release. Proc. Natl. Acad. Sci. U. S. A. 106, 21161-21166.

\section{NOTE ADDED AFTER ASAP PUBLICATION}

This paper originally posted on July 8, 2018. An additional Supporting Information file has been added. The revised version re-posted on August 17, 2018. 